\title{
Atuação de movimentos sociais e entidades na pandemia da COVID-19 no Brasil: 0 cuidado à pessoa idosa em Instituições de Longa Permanência
}

\author{
Performance of social movements and entities in the COVID-19 pandemic in Brazil: Older adults \\ care in long-term care facilities
}

\author{
Daiane de Souza Fernandes' 1 D \\ Roberta Cristina Cintra Taveira² ${ }^{(\mathbb{D}}$ \\ Luípa Michele Silva ${ }^{3}$ (D) \\ Luciana Kusumota ${ }^{4}$ ID \\ Karla Cristina Giacomin ${ }^{5} \mathbb{D}$ \\ Rosalina Aparecida Partezani Rodrigues 6 (D)
}

\section{Resumo}

Objetivo: analisar o conteúdo de documentos propostos por movimentos sociais e entidades de classe para orientar o cuidado em Instituições de Longa Permanência para Idosos (ILPI) durante a pandemia da COVID-19 no Brasil. Método: Pesquisa documental, retrospectiva, descritiva e exploratória. Foram identificados quatro websites de movimentos sociais e entidades e analisados seus respectivos documentos para orientar o cuidado à pessoa idosa que vive em ILPI no contexto da pandemia no Brasil. A análise foi do tipo temática, com auxílio do software IRaMuTeQ Resultados: Foram selecionados 28 textos para análise. O movimento social Frente Nacional de Fortalecimento à ILPI apresentou o maior quantitativo de produções. Na análise temática emergiram três classes: (1) O cuidado como estratégia de prevenção de contágio pela COVID-19 na ILPI; (2) O suporte dos gestores públicos enquanto direito da pessoa idosa que reside em ILPI; e (3) A preservação do vínculo sociofamiliar do residente da ILPI durante a pandemia. Conclusão: Os movimentos sociais e entidades tiveram um lépido desempenho no suporte às ILPI no Brasil por meio da divulgação de documentos que contribuíram para nortear o cuidado à pessoa idosa institucionalizada em situação de vulnerabilidade. Faz-se necessário maior envolvimento dos gestores públicos na proteção e direito à vida dessa população idosa.

1 Universidade de São Paulo (USP), Escola de Enfermagem de Ribeirão Preto, Programa de Pós-Graduação em Enfermagem Fundamental. Ribeirão Preto, SP, Brasil.

2 Universidade de São Paulo (USP), Faculdade de Medicina de Ribeirão Preto, Programa de Pós-Graduação em Nutrição e Metabolismo. Ribeirão Preto, SP, Brasil.

3 Universidade Federal de Catalão (UFCAT), Departamento de enfermagem, Unidade Acadêmica de Biotecnologia. Catalão, GO, Brasil.

4 Universidade de São Paulo (USP), Escola de Enfermagem de Ribeirão Preto, Departamento de Enfermagem Geral e Especializada, Programa de Pós-Graduação em Enfermagem Fundamental. Ribeirão Preto, SP, Brasil.

5 Secretaria Municipal de Saúde de Belo Horizonte, Coordenação de Atenção à Saúde da Pessoa Idosa. Belo Horizonte, MG, Brasil.

6 Universidade de São Paulo (USP), Escola de Enfermagem de Ribeirão Preto, Departamento de Enfermagem Geral e Especializada, Programa de Pós-Graduação em Enfermagem Fundamental. Ribeirão Preto, SP, Brasil.
Palavras-chave: Saúde do Idoso. Instituição de Longa Permanência para Idosos. Infecções por Coronavírus. Covid-19. Pandemias.

Os autores declaram não haver conflito na concepção deste trabalho.

Não houve financiamento para a execução deste trabalho. 


\section{Abstract}

Objective: To analyze the content of documents proposed by social movements and professional associations to guide care in Long-Term Care Institutions for the Elderly (LTCF) during a COVID-19 pandemic in Brazil. Method: This is a documentary, retrospective, descriptive and exploratory research. It was found four websites and their respective documents were identified to guide the care of the older adultswho live in LTCF in the context of the pandemic in Brazil. Thematic analysis was performed using IRaMuTeQ software. Results: 28 texts were selected for analysis. The social movement "National Front for Strengthening the LTCF" presented the highest number of productions. In the thematic analysis, three classes emerged: (1) Care as a contagion prevention strategy for COVID-19 at the LTCF; (2) The support of public managers as a right of the older adults living in the LTCF; and (3) The preservation of the social link of the LTCF residents during the pandemic. Conclusion: Social movements and organizations had a quick performance in supporting the LTCF in Brazil through the dissemination of documents that guided the care of institutionalized older adults in situations of vulnerability. Greater involvement of public managers in the protection and the right to life of these older adults population is necessary.

Keywords: Health of the Elderly. Homes for the Aged. Coronavirus Infections. Covid- 19. Pandemics.

\section{INTRODUÇÃO}

A COVID-19 é uma doença caracterizada por uma síndrome respiratória aguda grave causada por um vírus da família coronavírus, o SARS-CoV-2. Em virtude da gravidade e rápida disseminação mundial, foi reconhecida pela Organização Mundial da Saúde (OMS) como uma emergência de saúde pública, em janeiro de 2020, e em março do mesmo ano, como uma pandemia ${ }^{1}$.

A evolução da pandemia nos países asiáticos e europeus evidenciou o alto risco da doença para pessoas idosas, especialmente aquelas em condições frágeis de saúde e de extrema vulnerabilidade. Esse grupo apresenta a maior taxa de mortalidade quando comparado a outras faixas etárias ${ }^{2,3}$.

O cenário mostrou-se ainda mais crítico para pessoas residentes em Instituições de Longa Permanência para Idosos (ILPI). No Brasil, essas instituições podem ser públicas ou privadas, com a particularidade de funcionarem como uma residência para pessoas com 60 anos ou mais, independentemente de apresentarem ou não suporte familiar e são equipamentos da assistência social ${ }^{2}$.

Contudo, ainda é desconhecido o número exato de instituições com essa finalidade no país. De acordo com o Instituto de Pesquisa Econômica Aplicada (IPEA), em um levantamento nacional realizado em
2010, foram contabilizadas aproximadamente 3.548 instituições, 1.617 (45,6\%) delas de caráter filantrópico².

A população de pessoas idosas que mora em ILPI apresenta um conjunto de fatores que favorece a alta vulnerabilidade, tais como a idade avançada, presença de multimorbidades (hipertensão arterial, diabetes mellitus, cardiopatias, doenças pulmonares e outras), diferentes graus de dependência e a vivência em ambientes coletivos. Soma-se a essa problemática a inexistência de planos de enfrentamento no Brasil para proteção da pessoa idosa institucionalizada no decorrer da pandemia ${ }^{4,5}$.

Diante da urgência em criar medidas eficientes de contingência à COVID-19 nesses ambientes e da deficiência por parte do poder público na atenção a essa demanda, surgiram diferentes iniciativas por parte de organizações, entidades de classe e movimentos sociais. O objetivo principal foi subsidiar as instituições com informativos, protocolos, estratégias, relatórios, manifestos e cartas ${ }^{6}$.

Assim, algumas entidades e movimentos sociais brasileiros se organizaram e elaboraram diversos documentos com o intuito de nortear o cuidado prestado à população idosa institucionalizada. Tal atitude fica legitimada pela invisibilidade da implementação de políticas públicas no país voltadas para esse público. Assim, conhecer e analisar essa produção revela-se de fundamental importância para 
identificar os pontos frágeis que podem interferir no cuidado na ILPI, e fundamentalmente apontar intervenções necessárias para adoção de medidas preventivas contra a COVID-19 nestes espaços.

Como proposta para este estudo, foram elaboradas as seguintes questões de pesquisa: Quais os documentos elaborados pelos movimentos sociais e as entidades de classe na orientação do cuidado à pessoa idosa de ILPI durante a pandemia da COVID-19 no Brasil? Qual o conteúdo desses documentos?

O estudo apresenta como objetivo analisar o conteúdo de documentos propostos por movimentos sociais e entidades de classe para orientar o cuidado em ILPI durante a pandemia da COVID-19 no Brasil.

\section{MÉTODO}

Pesquisa documental, retrospectiva, descritiva e exploratória. A amostra foi constituída por documentos produzidos por movimentos sociais e entidades de classe para orientar o cuidado em ILPI durante a pandemia da COVID-19 no Brasil. Para seleção dos documentos, foram adotados os seguintes critérios de inclusão: divulgação de conteúdos como relatório técnico, protocolo, manifesto, plano de contingência, cartilha, informativo, capítulo de e-book, recomendações, plano de ação e carta que tinham como objetivo orientar o cuidado em ILPI; conteúdos disponíveis na íntegra nos seus respectivos websites e que apresentaram no seu interior as palavras-chaves: Instituição de Longa Permanência para Idosos, Idoso e COVID-19 (Coronavírus); documentos produzidos e divulgados no período de março à outubro de 2020.

A coleta de dados foi realizada no mês de outubro de 2020. A busca pelos dados foi pautada em três fases: pré-análise, exploração do material e análise dos dados. Na primeira, realizou-se o levantamento dos websites de movimentos sociais e entidades de classe, tendo como critérios de seleção: movimentos e entidades atuantes no Brasil, realizar a elaboração e divulgação de conteúdo sobre a pessoa idosa na pandemia da COVID-19 e sobre a orientação do cuidado ao residente em ILPI.

$\mathrm{Na}$ segunda etapa, houve o rastreio de todos os documentos elaborados. Na terceira etapa, fez-se a leitura minuciosa dos documentos encontrados, identificando-se os critérios de inclusão. $\mathrm{Na}$ amostra final 28 produções compuseram o banco de dados textual analisado. O fluxograma das etapas da coleta de dados está representado na (Figura 1).

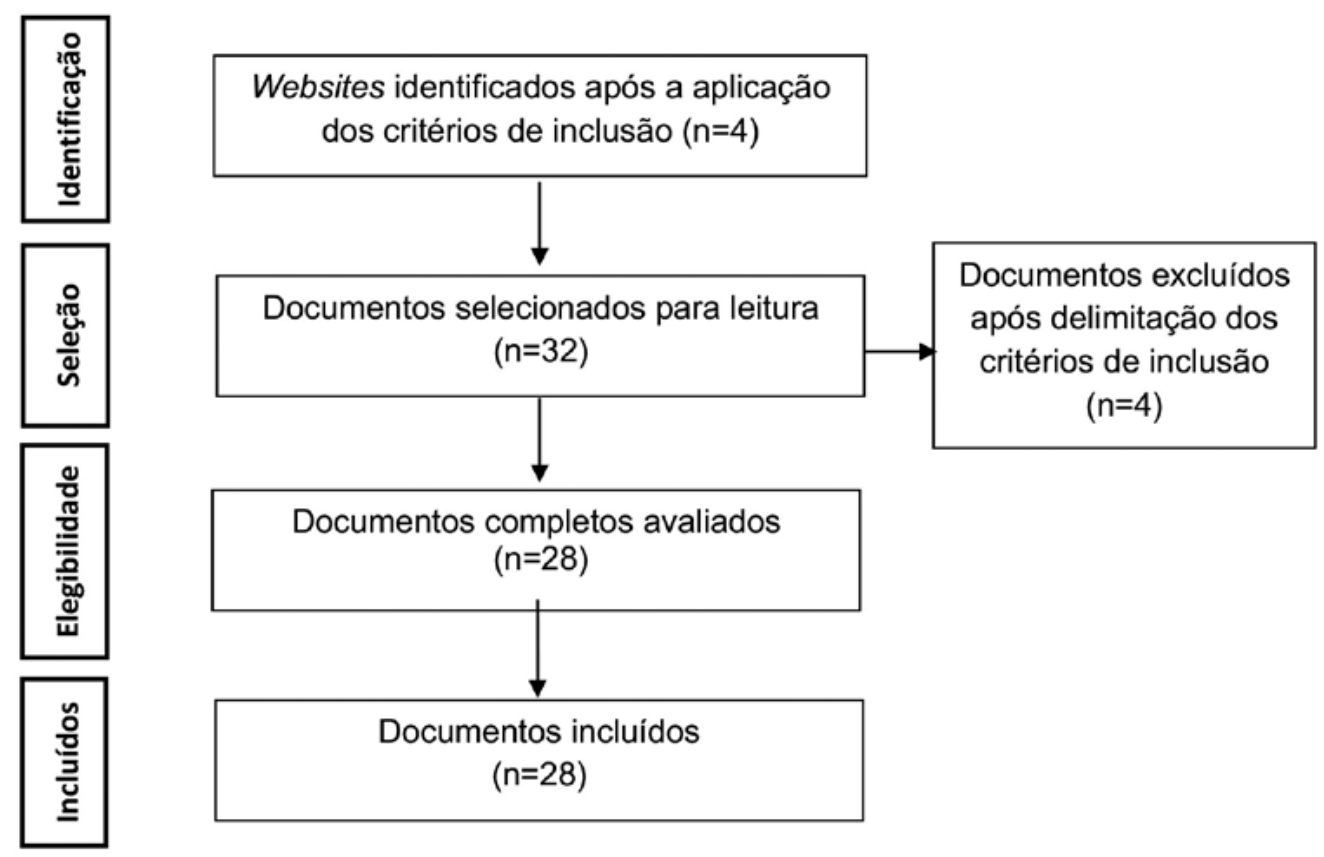

Figura 1. Fluxograma da seleção dos documentos utilizados no estudo. Ribeirão Preto, SP 2020. 
Os documentos foram organizados em um banco de dados, sendo possível identificar o movimento social ou entidade, título, tipo de documento, objetivos e conteúdo. Após a leitura minuciosa, obteve-se o banco de dados textual por meio do agrupamento dos textos em um só documento, criado no bloco de notas. Em seguida, este foi submetido ao processo de análise textual, com auxílio do software Interface de $\mathrm{R}$ pour les Analyses Multidimensionnelles de Textes et de Questionnaires - IRaMuTeQ, versão 0.6 alpha $3^{7}$.

Para auxiliar na análise, adotou-se como referencial a análise temática ${ }^{8}$, cujo processo consta de seis fases: (1) coleta dos dados - primeiro contato com o material; (2) criação de uma lista de ideias iniciais (códigos), momento em que o pesquisador procura a existência de temas; (3) classificação e agrupamento dos códigos em categorias; (4) criação dos temas, quando houve a independência de cada tema e foram obedecidos os princípios da homogeneidade interna e heterogeneidade externa, sendo esse um processo que elimina a ambiguidade no processo de distribuição temática; (5) atribuição de um nome a cada tema identificado; e, por fim, (6) a redação do relatório final ${ }^{8}$.

Para auxiliar nas etapas de três a cinco foi utilizado o software IRaMuTeQ com a escolha do método Reinert, que realiza uma classificação hierárquica de forma descendente. Tal classificação é definida por classes lexicais, em que cada uma delas representa um tema e pode ser descrito de acordo com as palavras que o definem e os respectivos segmentos de textos?. Para cada classe foram utilizados os segmentos obtidos na análise realizada pelo software e validados pelos pesquisadores.

$\mathrm{Na}$ análise temática emergiram três classes: a) $\mathrm{O}$ cuidado como estratégia de prevenção de contágio pela COVID 19 na ILPI; b) O suporte dos gestores públicos enquanto direito da pessoa idosa que reside em ILPI; e c) A preservação do vínculo sociofamiliar do residente da ILPI durante a pandemia.

Também foi utilizada a Análise de Similitude, que cria um gráfico de semelhança, representando os elos entre as formas analisadas. Para elaboração do gráfico, os parâmetros utilizados na análise foram as palavras ativas e com frequência maior do que 35 (f $\geq 35$ ), para visualização dos termos mais frequentes, pois com uma frequência mais baixa, a imagem fica com o aspecto poluído, dificultando a compreensão do leitor?

Com base no relatório da análise e nos gráficos gerados, os dados foram analisados à luz da literatura pertinente conforme a técnica de análise de conteúdo temática.

Por ter utilizado informações de acesso público e gratuito, disponibilizadas nos websites dos movimentos sociais e entidades de classe, a presente pesquisa não foi submetida ao Comitê de Ética, conforme a Resolução do Conselho Nacional de Saúde $n^{\circ}$ $510 / 2016$.

Além dos aspectos éticos, as etapas da pesquisa e elaboração do artigo responderam aos critérios da Consolidated criteria for reporting qualitative research ${ }^{10}$.

\section{RESULTADOS}

Dentre os movimentos sociais e entidades selecionados para analisar os documentos produzidos, a Frente Nacional de Fortalecimento à ILPI (FNILPI) ${ }^{11}$ apresentou o maior quantitativo de documentos, totalizando 12 sendo 2 (dois) relatórios, 6 (seis) cartilhas, 1 (um) protocolo, 1 (um) manifesto, 1 (um) informativo e 1 (um) plano de contingência. O Departamento Científico de Enfermagem Gerontológica da Associação Brasileira de Enfermagem (DCEG) ${ }^{12}$ apresentou 10, sendo 1 (um) informativo e 9 (nove) capítulos de livros. A Sociedade Brasileira de Geriatria e Gerontologia (SBGG) ${ }^{13}$ divulgou 3 (três), por meio de recomendações, e a Aliança Voluntária para Proteção dos Idosos Institucionalizados (ILPI.me) ${ }^{14}$ totalizou 3 (três) documentos, sendo 1 (um) plano de ação, 1 (um) protocolo e 1 (uma) carta.

$\mathrm{Na}$ análise dos dados, o banco textual permitiu ao software IRaMuTeQ formar 154 segmentos de texto, que possibilitaram identificar 1.002 ocorrências de termos, dos quais 332 palavras foram selecionadas. Destas, foram identificadas 190 palavras diferentes, com uma única ocorrência, correspondendo a $57,23 \%$ do total de palavras analisadas e a $18,96 \%$ das ocorrências encontradas. 
Quanto ao conteúdo dos documentos analisados, de acordo com a Figura 2, os termos mais recorrentes utilizando uma frequência de palavras igual ou maior que 35 foram: idoso ( $\mathrm{f}=475)$; pessoa ( $\mathrm{f}=250)$; ILPI $(\mathrm{f}=216)$; saúde $(\mathrm{f}=190)$; cuidado $(\mathrm{f}=169)$; profissional ( $f=138)$; social ( $f=127)$; público $(f=114)$; direito ( $\mathrm{f}=111)$; ação $(\mathrm{f}=100)$; equipamentos $(\mathrm{f}=98)$; familiar $(\mathrm{f}=96)$; pandemia $(\mathrm{f}=96)$; recurso $(\mathrm{f}=92)$; assistência $(\mathrm{f}=76)$; Conselho $(\mathrm{f}=72)$; considerar $(\mathrm{f}=72)$; vida $(\mathrm{f}=71)$; prevenção $(\mathrm{f}=70)$; proteção $(\mathrm{f}=69)$; serviço $(\mathrm{f}=69)$; necessidade $(\mathrm{f}=66)$; necessário $(\mathrm{f}=66)$; plano $(\mathrm{f}=52)$; isolamento $(\mathrm{f}=52)$.

A Figura 3 representa a classificação hierárquica descendente, com a organização em classes com nuvem de palavras. As classes 1 e 2 compõem um mesmo subcorpus (58,9\%), sendo que na Classe 1 encontram-se $46,4 \%$ dos segmentos de texto e na Classe 2, 12,5\% dos segmentos de textos. Na classe 1, as palavras mais frequentes foram: ILPI; COVID; cuidado; profissional; caso; equipamento; prevenção; suspeito. $\mathrm{Na}$ classe 2, destacaram-se: familiar; rede; contato; cognitivo; suporte; virtual; querido; incentivar. A classe 3 apresenta o outro subcorpus com 41,1\% dos segmentos de texto, tendo como ênfase: público; social; direito; recurso; assistência; nacional; Conselho.

As classes obtidas na análise e alguns trechos extraídos dos documentos elaborados pelos movimentos e entidades de classe estão representados no Quadro 1.

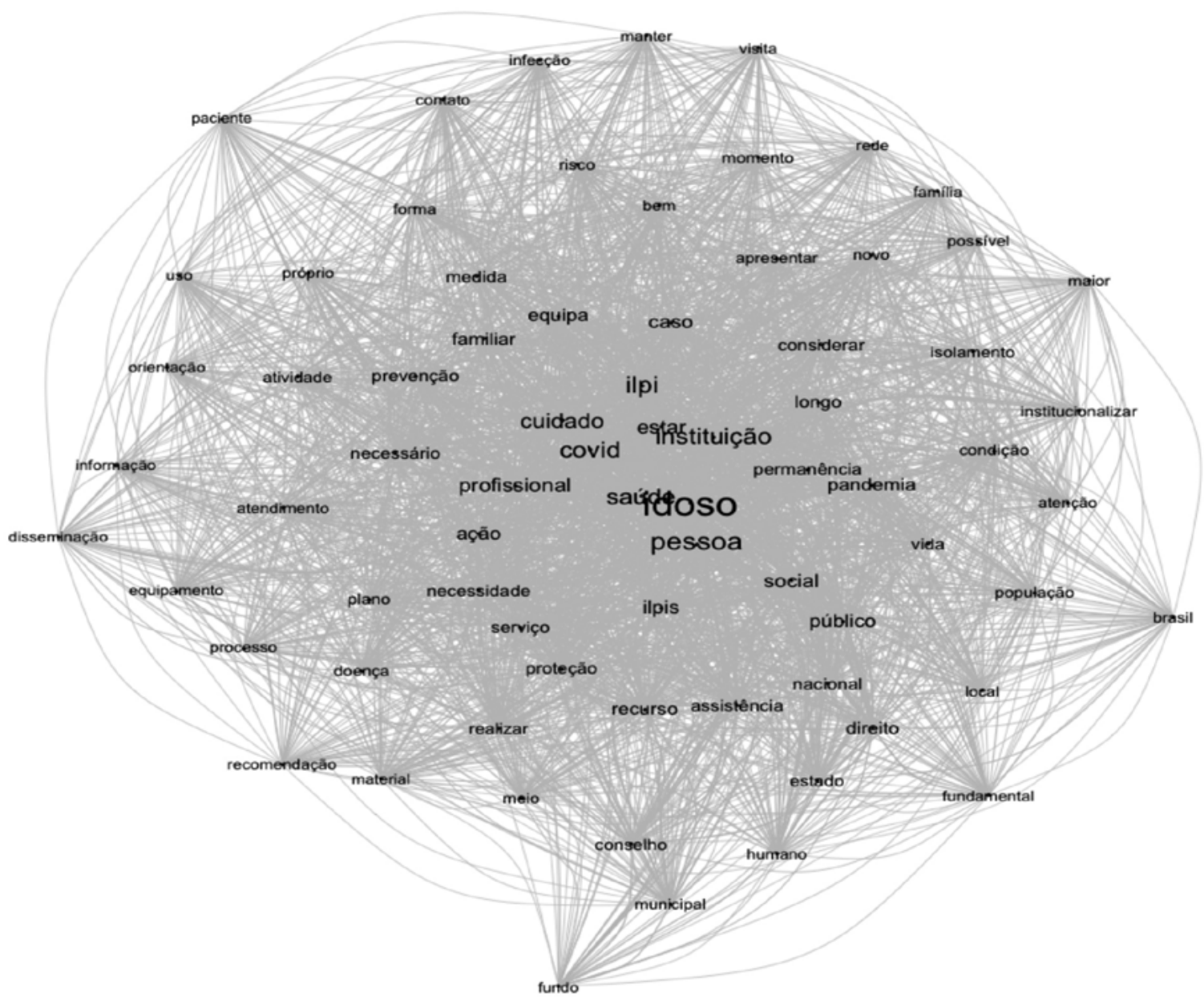

Figura 2. Gráfico de similitude em formato de rede com as palavras que apresentaram conexão nos documentos analisados. Ribeirão Preto, SP 2020. 


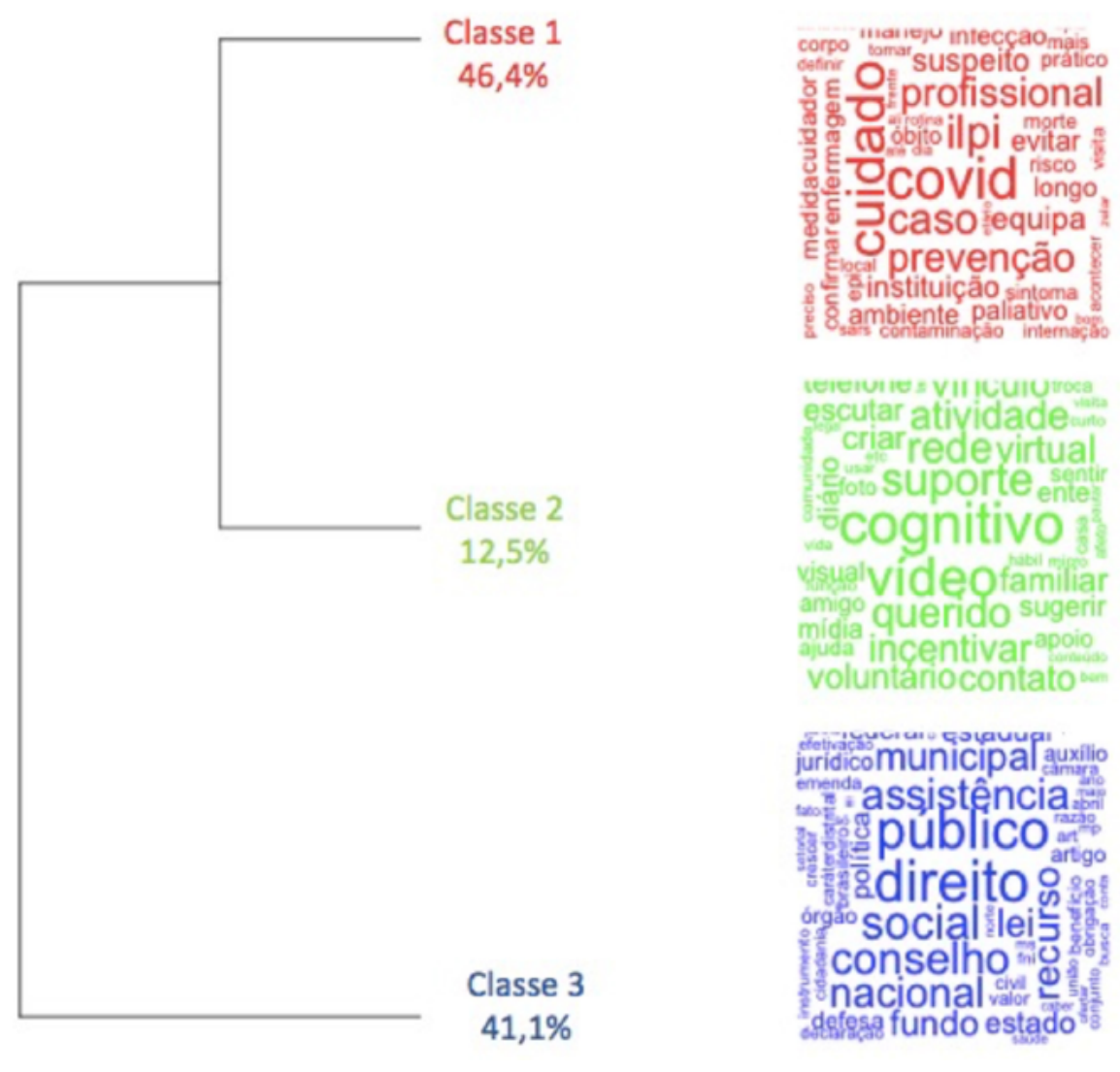

Figura 3. Dendograma das classes fornecidas pelo IRAMUTEQ em formato de nuvem de palavras a partir dos documentos analisados que envolveram a temática de orientações para o cuidado do idoso na ILPI durante a pandemia da COVID-19. Ribeirão Preto, SP, 2020.

Quadro 1. Apresentação das classes e alguns trechos extraídos dos documentos analisados. Ribeirão Preto, SP, 2020.

\begin{tabular}{|c|c|}
\hline Classe & Trecho dos documentos analisados \\
\hline \multirow[t]{3}{*}{$\begin{array}{l}\text { O cuidado como estratégia de } \\
\text { prevenção de contágio pela } \\
\text { COVID-19 na ILPI }\end{array}$} & $\begin{array}{l}\text { "Os gestores das ILPIs devem certificar-se de que os profissionais das ILPI e de apoio foram } \\
\text { capacitados e tenham praticado o uso apropriado dos Equipamentos de Proteção Individual EPIs } \\
\text { antes de iniciar o atendimento as pessoas idosas na unidade incluindo os serviços prestados em caso } \\
\text { suspeito ou confirmado de infecção pelo novo Coronavirus [...]" (FNILPI 01) }\end{array}$ \\
\hline & $\begin{array}{l}\text { "Organizar planos de ação para prevenção e manejo de idosos moradores, promover treinamento } \\
\text { da equipe assistencial para as medidas programadas, reforçar condutas de bigiene do local e dos } \\
\text { protocolos de bigiene de mãos e proteção dos moradores e funcionários [...]" (SBGG 02) }\end{array}$ \\
\hline & $\begin{array}{l}\text { "Apresentar estratégias e orientações para as equipes de profissionais atuantes na ILPI sobre a } \\
\text { prevenção da COVID-19 [...]" (ILPI.me 02) }\end{array}$ \\
\hline \multirow{3}{*}{$\begin{array}{l}\text { O suporte dos gestores públicos } \\
\text { enquanto direito da pessoa } \\
\text { idosa que reside em ILPI }\end{array}$} & $\begin{array}{l}\text { "As ILPIs são locais de moradia e equipamentos da assistência social não tendo em sua maioria } \\
\text { estrutura ou recursos humanos para oferecer cuidados especificos de saúde [...]" (DCEG 08) }\end{array}$ \\
\hline & $\begin{array}{l}\text { "Em todo o mundo a face mais cruel da pandemia se revela no abandono das pessoas idosas, } \\
\text { na falta de acesso a recursos na saúde com elevada mortalidade daquelas que vivem em abrigos e } \\
\text { instituiçoes [...]" (SBGG 03) }\end{array}$ \\
\hline & $\begin{array}{l}\text { "As ILPIs representam o local de moradia das pessoas idosas e são equipamentos da assistência } \\
\text { social não tendo portanto estrutura ou recursos humanos para oferecer cuidados específicos de saúde } \\
\text { a seus residentes [...]" (ILPI.me 01) }\end{array}$ \\
\hline
\end{tabular}


Continuação do Quadro 1

\begin{tabular}{|c|c|}
\hline Classe & Trecho dos documentos analisados \\
\hline \multirow[t]{3}{*}{$\begin{array}{l}\text { A preservação do vínculo } \\
\text { sociofamiliar do residente da } \\
\text { ILPI durante a pandemia }\end{array}$} & $\begin{array}{l}\text { "Apoiar as familias com idosos institucionalizados, manter um canal de comunicação regular com } \\
\text { a rede sociofamiliar, favorecer o contato virtual das famílias e amigos com os residentes, comunicar } \\
\text { caso haja casos suspeitos ou confirmados de Covid } 19 \text { na ILPI [...]" (FNILPI 03) }\end{array}$ \\
\hline & $\begin{array}{l}\text { "É extremamente importante que os familiares sejam comunicados de forma adequada, um } \\
\text { contato bem feito evitará mal entendidos, trará os familiares como aliados na proteção dos idosos, } \\
\text { minimizará indignações contra medidas restritivas, trará um caminho prático e claro para orientar } \\
\text { os comportamentos [...]"(ILPI.me 2) }\end{array}$ \\
\hline & $\begin{array}{l}\text { "Utilizar ferramentas digitais com boa conectividade para o contato dos idosos com seus familiares } \\
\text { com ou sem capacidade cognitiva implementando horários especificos para as visitas virtuais, após, } \\
\text { realizar higienização dos tablets ou celulares [...]"(DCEG 01) }\end{array}$ \\
\hline
\end{tabular}

Frente Nacional de Fortalecimento à ILPI (FNILPI); Sociedade Brasileira de Geriatria e Gerontologia (SBGG); Aliança Voluntária para Proteção dos Idosos Institucionalizados (ILPI. me); Departamento Científico de Enfermagem Gerontológica (DCEG).

\section{DISCUSSÃO}

Os resultados foram fundamentados na análise, sendo possível identificar classes que emergiram do teor dos documentos. Foram verificadas três vertentes com intrínseca relação ao grau de importância da pessoa idosa que reside na ILPI: cuidado, suporte do poder público e manutenção do vínculo sociofamiliar. Tal constatação denota uma inquietude dos movimentos e entidades em relação à necessidade de proteção, acolhimento e defesa de direitos dessa população para orientar um melhor cuidado.

Os documentos analisados expressam a importância da transmutação do olhar para as pessoas idosas que residem em ILPI, pois apresentam um perfil de envelhecimento acompanhado de vulnerabilidades que demandam efetivação de políticas públicas urgentes.

Importante reconhecer que a Classe 1 , que trata da importância do cuidado direcionado como estratégia de prevenção da COVID 19 entre os residentes das instituições, e a Classe 2, que aborda o cuidado como direito, compõem um mesmo subcorpus. Assim, infere-se a necessidade de interação entre os cuidados propostos e o desejável suporte da gestão pública para a execução desses cuidados.

No Brasil, a ILPI enquadra-se como um local de moradia, estando diretamente vinculada ao Sistema Único de Assistência Social ${ }^{5,15}$. Contudo, este ambiente pode ser desprovido de estrutura física e de recursos humanos adequados para a prestação de um cuidado integral ${ }^{2}$. Essa característica viuse agravada durante a pandemia devido à alta vulnerabilidade dos residentes para contraírem a infecção e desenvolverem complicações ${ }^{16-20}$, em virtude da fragilidade, maior grau de dependência associada à redução da reserva fisiológica, perda da capacidade física e menor reação positiva face às adversidades ${ }^{21}$.

Além disso, na prática, muitas ILPI apresentam dificuldades para seguir as recomendações de higiene preconizadas para interromper a transmissã $\mathrm{O}^{22}$, o que demanda a adoção de medidas urgentes para contenção da disseminação do vírus nesses espaços.

Dessa forma, a adoção de estratégias eficazes de prevenção para evitar a propagação da infecção nestes locais inclui: a organização do espaço para evitar contato muito próximo; a restrição de visitas; as orientações para os colaboradores sobre a importância de medidas simples como lavagem regular das mãos e/ou utilização de álcool 70\%, bem como a troca de roupas e calçados ao chegar na instituição; a reconfiguração de atividades realizadas em grupos; a sistematizada manutenção da limpeza do local; além da identificação precoce de residentes sintomáticos ${ }^{17,18,23,24}$. Destaca-se que, dentre essas medidas, a identificação precoce dos sintomas gripais e suas nuances revela-se primordial, pois pessoas idosas fragilizadas podem apresentar sintomas atípicos incluindo a ausência de febre, tosse ou produção de expectoração ${ }^{16}$. Assim, faz-se 
necessária a capacitação de cuidadores e equipe da ILPI para uma intervenção prévia com intuito de proteção dos residentes.

A ILPI, sendo considerada um local de residência, não possui condições estruturais para fornecer cuidados de casos descompensados de COVID-19, pois não foi projetada para tais situações e os cuidadores e equipe não foram capacitados para intervir em casos de doenças respiratórias graves. Porém, denota-se a importância da sua atuação em conjunto com a rede de saúde para prestar o cuidado integralizado aos seus residentes ${ }^{22}$.

O movimento social da Frente Nacional de Fortalecimento à ILPI propôs ainda um documento para a intervenção do cuidado à pessoa idosa na ILPI, no manual de Boas Práticas ${ }^{15}$. O documentou considerou o risco de contaminação dos residentes relacionado à atuação de cuidadores, membros da equipe, visitas, entregadores de insumos e até mesmo dos próprios residentes que podem ter entrado em contato com pessoas contaminadas dentro da instituição $0^{15,19}$.

A adoção de estratégias para educação permanente dos profissionais que atuam na ILPI deve ser implementada, utilizando a disseminação de conhecimento e adoção de medidas preventivas a partir de protocolos e normas oficiais. Necessário que essas ações integrem as áreas da saúde, assistência social, trabalho e direitos humanos ${ }^{15}$.

A pandemia sinalizou a necessidade, em um futuro próximo, de um olhar mais direcionado em relação ao cuidado na ILPI, com o estabelecimento de modelos de cuidados integrados, envolvendo também a rede de vínculo sociofamiliar ${ }^{17}$.

A Classe 2 enfocou a importância da atuação do poder público na garantia do direito social e da proteção à vida da pessoa idosa na pandemia. Em países do hemisfério norte, um alto índice de óbitos foi registrado em ILPI, refletindo a vulnerabilidade dos residentes que vivem nesses locais, e ainda denotando uma fragilidade das políticas públicas com a não inclusão das ILPI no planejamento sistemático de resposta à COVID-19. Importante ressaltar que a falta de efetivação de políticas públicas para esse grupo é uma questão de longa data, com debilidades relacionadas a financiamento, deficit de recursos humanos e equipe com conhecimentos diminutos em gerontologia e cuidados a longo prazo ${ }^{3,19}$.

Além disso, o momento inusual vivenciado nesses espaços vem requerendo dos colaboradores da instituição, principalmente cuidadores, a oferta de um melhor cuidado possível. Na prática, essas mudanças repercutem no aumento da carga trabalho em decorrência da adoção criteriosa de medidas preventivas e da necessidade de reinvenção na prestação de um cuidado seguro, dada a escassez de equipamentos de proteção individual e de outros suprimentos primordiais para a qualidade no atendimento ${ }^{19,25,26}$. Este momento incita o impulsionamento para mudanças em políticas e diretrizes relacionadas à saúde, podendo promover uma readequação e ser disseminada de forma eficiente $^{16}$, principalmente para as pessoas idosas institucionalizadas.

Nesse sentido, medidas emergenciais deverão ser implementadas pelos Estados e Municípios, inclusive com cofinanciamento federal. O poder público deve oferecer ações de proteção à vida, ofertar auxílios em bens materiais e valores financeiros em situações provisórias no contexto de calamidade pública, requerendo investimento nas ILPI na pandemia ${ }^{27}$.

A elaboração de planos de contingência para responder às necessidades que emergiram em virtude da pandemia deve ser adaptada às características e necessidades de cada ILPI. Isso demanda uma articulação factual com a atenção primária à saúde atuando em conjunto com a equipe da instituição, de modo a propiciar um acompanhamento que possibilitará melhor qualidade de vida e controle da disseminação do vírus. Contudo, não se mostrará uma proposta viável se não houver alocação e/ou organização de recursos para essa finalidade ${ }^{28}$.

Outro impedimento para a efetivação de políticas públicas nesses locais é a falta de integração entre os serviços sociais e de saúde ${ }^{28}$. As dificuldades na disponibilização de cuidados de longa duração estão alicerçadas na falta de integração de paradigmas de cuidados de saúde pautados nas necessidades individuais, bem como na ausência de integração dos 
cuidados de saúde e sociais ${ }^{17}$. A associação efetiva entre ambas as esferas refletirá na disponibilização de um modelo de cuidado integrado e condizente com as necessidades dessas instituições, a exemplo do observado em países desenvolvidos.

Embora a discussão sobre estes espaços ainda seja tímida, principalmente em se tratando de posições governamentais, reflexões e ações imediatas são necessárias, visto que esses locais, por serem mais vulneráveis para disseminação do vírus, requerem apoio e ações urgentes de proteção ao infortúnio ${ }^{21}$. Ademais, dados indicam que os efeitos advindos da pandemia persistirão mesmo após a sua finalização, com repercussões graves na economia e na saúde ${ }^{29}$. Ratifica-se, portanto, a necessidade de os gestores políticos priorizarem a crise no atendimento às pessoas idosas de ILPI e de estabelecer modelos de cuidados integrados, envolvendo também a rede de vínculo sociofamiliar ${ }^{17}$, como apontado na Classe 3.

A Classe 3 representou a importância na manutenção do vínculo sociofamiliar como estratégia minimizadora dos efeitos advindos do isolamento social, condição muitas vezes contumaz no cotidiano do residente da ILPI. A restrição de visitas foi uma das primeiras medidas adotadas para minimizar os riscos de contaminaçãa ${ }^{17,19,20,25,26}$. No entanto, essa determinação acarretou sofrimento e repercutiu na saúde mental das pessoas idosas ${ }^{29}$, em muitos casos desencadeando casos de depressão e ansiedade, sendo mais evidentes em pessoas idosas com quadro demencial $^{25}$.

O isolamento social entre as pessoas idosas configura-se como um problema de saúde pública devido aos riscos elevados de complicações neurológicas, cardiovasculares, autoimunes, além de comprometimentos cognitivos e de saúde mental ${ }^{30}$. Tais condições mostram-se ainda mais evidentes entre residentes de ILPI, os quais já vivem socialmente isolados ${ }^{22} \mathrm{e}$ apresentam oportunidades reduzidas de comunicação com outras pessoas, espaços e no envolvimento de atividades sociais que apresentam significado particular. Com isso, urge a necessidade de suporte e adaptação às necessidades de comunicação com o intuito de auxiliar a tomada de decisão e, consequentemente, melhorar a qualidade de vida e o bem-estar dessa população $0^{30}$.
Torna-se imprescindível que as pessoas idosas sejam informadas sobre as ameaças à sua saúde e sobre os procedimentos que visam à segurança e proteção para que possam aderir ao novo momento demandado pela pandemia ${ }^{17}$. Premente também repensar estratégias para manutenção dos vínculos sociofamiliares neste momento.

A utilização das tecnologias on-line é um mecanismo de suporte social, manutenção da rede de apoio e sentimento de pertencimento ${ }^{30}$. As intervenções podem ser simples e envolver visitas virtuais, envio de cartas, ligações telefônicas, videochamadas, troca de fotos, dentre outras estratégias ${ }^{19,23,31}$ realizadas com pessoas significativas, família, amigos e organizações voluntárias que prestam serviço na ILPI. Por outro lado, a família do residente requer segurança para a prevenção da COVID-19, mas também o diálogo com a pessoa idosa e a equipe para transmitir segurança para o cuidado do seu familiar que vive na ILPI.

Em momentos de emergências de saúde, a população idosa é a parte invisível da crise e, fazendo referência à pessoa idosa que vive em ILPI, essa característica requer a adoção de estratégias para assegurar a vida dessas pessoas. A realização de campanhas de informação à sociedade deve ser direcionada para proteger os mais vulneráveis, tornando-os visíveis para o corpo social ${ }^{32}$.

Neste período, houve uma rápida irrupção de informações relacionadas ao cuidado nesses ambientes $^{31}$. No Brasil, o Ministério da Saúde divulgou notas técnicas ${ }^{33-35}$ com orientações para prevenção e controle de infecções pelo novo coronavírus em ILPI. Contudo, iniciativas realizadas por movimentos e entidades como a Frente Nacional de Fortalecimento à ILPI, o Departamento Científico de Enfermagem Gerontológica, a Sociedade Brasileira de Geriatria e Gerontologia e a Aliança Voluntária para Proteção dos Idosos Institucionalizados reforçaram e favoreceram a divulgação de informações por meio de documentos, orientações para o cuidado e adoção de melhores práticas para controle da infecção. Todas essas iniciativas contribuem para a defesa das pessoas idosas que vivem em ILPI e para a visibilidade deste coletivo para a sociedade. 
Identifica-se como limitação deste estudo a seleção dos principais movimentos sociais e entidades que disponibilizaram conteúdos on-line, não abrangendo possíveis publicações em outros formatos de divulgação.

\section{CONCLUSÃO}

A pandemia acarretou óbitos de pessoas idosas em várias ILPI no mundo, dada a alta vulnerabilidade de seus residentes para infecção e complicações do vírus. Os movimentos sociais e entidades tiveram uma célere dinâmica no apoio às ILPI por meio da elaboração e divulgação de documentos para orientar o cuidado prestado nessas instituições no contexto de pandemia da COVID-19. Apontaram a urgente necessidade na adoção de medidas preventivas para controle da disseminação do vírus nesses locais.

Os documentos analisados também enfatizaram a necessidade de orientação sobre um cuidado direcionado para prevenção da COVID-19 com enfoque na capacitação da equipe, na importância da atuação do poder público no suporte a esses locais, ainda esquecidos e não valorizados, com cofinanciamentos para garantia de materiais e equipamentos para um cuidado de qualidade e da manutenção da rede de apoio das pessoas idosas. Essas medidas foram destacadas tendo em vista os grandes impactos na saúde mental e na qualidade de vida de todos os envolvidos.

Editado por: Maria Luiza Diniz de Sousa Lopes

\section{REFERÊNCIAS}

1. World Health Organization. Prevención y control de infecciones en los centros de atención de larga estancia en el contexto de la COVID-19 [Internet]. Geneva: WHO; 2020 [acesso em 20 nov. 2020]; [6 telas]. Disponível em: https://apps.who.int/ iris/bitstream/handle/10665/331643/WHO2019-nCoV-IPC_long_term_care-2020.1-spa. pdf? sequence $=1 \&$ is Allowed $=\mathrm{y}$

2. Watanabe HAW, Domingues MARC, Duarte YAO. COVID-19 and homes for the aged: care or an announced death? Geriatr Gerontol Aging. 2020;14(2):143-5. Disponível em: https://doi. org/10.5327/Z2447-2123202020142LTTR

3. O’Neill D, Briggs R, Holmerová I, Samulelsson O, Gordon AL, Martin FC. COVID-19 highlights the need for universal adoption of standards of medical care for physicians in nursing homes in Europe. Eur Geriatr Med. 2020;11(4):645-50. Disponível em: https://doi.org/10.1007/s41999-020-00347-6

4. Kalache A, Silva A, Giacomin KC, Lima KC, Ramos LR, Louvison M, et al. Aging and inequalities: social protection policies for older adults resulting from the Covid-19 pandemic in Brazil. Rev Bras Geriatr Gerontol. 2020;23(6)1-10. Disponível em: https://doi. org/10.1590/1981-22562020023.200122

5. Menezes TMO, Freitas AVS, Pedreira LC, Amaral JB. Telemonitoring of Brazilian Nursing homes before Coronavirus and COVID-19 Infections. Rev Bras Enferm. 2020;73(Suppl 2):e20200350. Disponível em: https://doi.org/10.1590/0034-7167-2020-0350

6. Wachholz PA, Moreira GV, Oliveira D, Watanabe HAW, Boas JFV. Ocorrências de infecções e mortalidade por COVID-19 em residenciais para idosos no Brasil. Scielo Preprints [Preprint]. 2020 [Postado 03 ago. 2020; acesso em 05 out. 2020]:15 p. Disponível em: https://doi.org/10.1590/ SciELOPreprints.1032

7. Ratinaud P. IRaMuTeQ: Interface de R pour les analyses multidimensionnelles de textes et de questionnaires [Internet]. [Sem local]: IRAMUTEQ; 2020 [acesso em 10 out. 2020]. Disponível em: http:// www.iramuteq.org

8. Braun V, Clarke V. Using thematic analysis in psychology. Qual Res Psychol. 2006;3(2):77-101. Disponível em: http://dx.doi. org/10.1191/1478088706qp063oa

9. Reinert M. Alceste, une méthodologie d'analyse des données textuelles et une application: Aurelia de Gerard de Nerval. Bull Methodol Sociol. 1990;26:2454. Disponível em: https://doi.org/10.1177/07591063 9002600103

10. Tong A, Sainsbury P, Craig J. Consolidated criteria for reporting qualitative research (COREQ): a 32item checklist for interviews and focus groups. Int J Qual Health Care. 2007;19(6):349-57. Disponível em: http://dx.doi.org/10.1093/intqhe/mzm042

11. Frente Nacional de Fortalecimento à ILPI [Internet]. [Sem local]; 2020 [acesso em 05 out. 2020]. Disponível em: https://www.frentenacionalilpi.com.br/ 
12. Associação Brasileira de Enfermagem, Departamento Científico de Enfermagem Gerontológica [Internet]. Brasília, DF: ABEN; 2020 [acesso em 05 out. 2020]. Disponível em: http://www.abennacional.org.br/site/ enfermagem-gerontologica/

13. Sociedade Brasileira de Geriatria e Gerontologia [Internet]. Rio de Janeiro: SBGG; 2020 [acesso em 06 out. 2020]. Disponível em: https://sbgg.org.br/ category/covid-19/

14. Aliança Voluntária para Proteção dos Idosos Institucionalizados - ILPI. me [Internet]. 2020 [acesso em 07 out. 2020]. Disponível em: https://www.ilpi.me/

15. Frente Nacional de Fortalecimento à Instituição de Longa Permanência para Idoso. Boas práticas para as Instituições de Longa Permanência para Idosos no enfrentamento da pandemia de Covid-19: estratégias e protocolos [Internet]. Rosalina Partezani Rodrigues, coord. Brasília, DF: FN-ILPI; 2020. [acesso em 01 out. 2020]. Disponível em: https://894d0f6a-a0f84238-b884-a5387e68c8c7.filesusr.com/ugd/2bdc6d_ e984b4931d964a53a55a4a3363bc8ca8.pdf

16. D'Adamo H, Yoshikawa T, Ouslander JG. Coronavirus Disease 2019 in Geriatrics and LongTerm Care: the ABCDs of COVID-19. J Am Geriatr. 2020;68:912-7. Disponível em: htpps://doi. org/10.1111/jgs.16445

17. Roller-Wirnsberger R, Lindner S, Matijevic A, Stolz E, Wirnsberger G. Nursing home care in Austria during SARS-COVID-19 pandemia 2020. J Nurs Home Res. 2020;6:50-2. Disponível em: http://dx.doi. org $/ 10.14283 /$ jnhrs. 2020.14

18. Moraes EN, Viana LG, Resende LMH, Vasconcellos LS, Moura AS, Menezes A. COVID-19 in long-term care facilities for the elderly: laboratory screening and disease dissemination prevention strategies. Ciênc Saúde Colet. 2020;25(9):3445-58. Disponível em: https://doi.org/10.1590/1413-81232020259.20382020.

19. Chee SY. COVID-19 Pandemic: the Lived Experiences of Older Adults in Aged Care Homes. Millen Asia. 2020;11(3):299-317. Disponível em: https://doi.org/10.1177/0976399620958326

20. Kunz R, Minder M. COVID-19 pandemic: palliative care for elderly and frail patients at home and in residential and nursing homes. Swiss Med Wkly. 2020;150:w20235. Disponível em: https://doi. org/10.4414/smw.2020.20235

21. Tarazona-Santabalbina FJ, Martínez-Velilla N, Vidán MT, García-Navarro JA. COVID-19, older adults and ageism: Mistakes that should never happen again. Rev Esp Geriatr Gerontol. 2020;55(4):191-2. Disponível em: https://doi.org/10.1016/j.regg.2020.04.001
22. Gardner W, States D, Bagley N. The Coronavirus and the Risks to the Elderly in Long-Term Care. J Aging Soc Policy. 2020;32:4-5: 310-5. Disponível em: https://doi.org/10.1080/08959420.2020.1750543

23. Associação Brasileira de Enfermagem, Departamento Científico de Enfermagem Gerontológica. Comunicação aos trabalhadores de enfermagem das instituições de longa permanência de idosos (ILPI) para o enfrentamento da disseminação da COVID-19 [Internet]. ABEN; 2020 [acesso em 20 nov. 2020]. Disponível em: http://www.abennacional.org.br/ site/wp-content/uploads/2020/03/DCEG-ABEn_ Informe_COVID-19-ILPI.pdf

24. Sociedade Brasileira de Geriatria e Gerontologia. Recomendações para Prevenção e Controle de infecções por coronavírus (SARS-Cov-2) em Instituições de Longa Permanência para Idosos (ILPIs) [Internet]. Rio de Janeiro: SBGG; 2020 [acesso em 01 out. 2020]. Disponível em: https://sbgg. org.br/recomendacoes-para-prevencao-e-controlede-infeccoes-por-coronavirus-sars-cov-2-eminstituicoes-de-longa-permanencia-para-idosos-ilpis/

25. El Haj M, Altintas E, Chapelet G, Kapogiannis D, Gallouj K. High depression and anxiety in people with Alzheimer's disease living in retirement homes during the covid-19 crisis. Psychiatry Res. 2020;291:110. Disponível em: https://doi.org/10.1016/j. psychres.2020.113294

26. American Geriatrics Society. American Geriatrics Society (AGS) policy brief: COVID-19 and assisted living facilities. J Am Geriatr Soc. 2020;68(5):908-11. Disponível em: https://doi-org.ez67.periodicos.capes. gov.br/10.1111/jgs.16510

27. Frente Nacional de Fortalecimento à Instituição de Longa Permanência para Idoso. Instituições de Longa Permanência para Idosos e o enfrentamento da pandemia de Covid-19: subsídios para a Comissão de Defesa dos Direitos do Idoso da Câmara Federal: relatório técnico [Internet]. Karla Cristina Giacomin, org. Brasília, DF: FN-ILPI; 2020 [acesso em 01 out. 2020]. Disponível em: https://894d0f6a-a0f8-4238b884-a5387e68c8c7.filesusr.com/ugd/2bdc6d_ fec825b075a4457dbd6c6f4d6a9e5e26.pdf

28. Blanco-Tarrio E, Blanco Sánchez G. Primary care, residential homes for the elderly, and COVID-19. Semergen. 2020;46 Suppl 1:26-34. Disponível em: https://doi.org/10.1016/j.semerg.2020.06.003

29. Hall G, Deepika R, Laddu SA, Phillips CJ, Lavie RA. A tale of two pandemics: How will COVID-19 and global trends in physical inactivity and sedentary behavior affect one another? Progr Cardiovas Dis. 2020;64:108-10. Disponível em:https://doi. org/10.1016/j.pcad.2020.04.005 
30. Armitage R, Nellums LB. COVID-19 and the consequences of isolating the elderly. Lancet Public Health. 2020;5(5):e256. Disponível em: https://doi. org/10.1016/S2468-2667(20)30061-X

31. Moser A, Pariser P, Verma A, Razak F, Wong B, Pus L, et al. COVID-19 in LTC: reflections from Ontario Canada. J Nurs Home Res. 2020;6:45-6. Disponível em: http://dx.doi.org/10.14283/jnhrs.2020.12

32. Etard J-F, Vanhems P, Atlani-Duault L, Ecochard $R$. Potential lethal outbreak of coronavirus disease (COVID-19) among the elderly in retirement homes and long-term facilities. Euro Surveill. 2020;25(15):110. Disponível em: https://doi.org/10.2807/15607917.ES.2020.25.15.2000448

33. Brasil. Agência Nacional de Vigilância Sanitária. Orientações para a prevenção e o controle de infecções pelo Novo Coronavírus em Instituições de Longa Permanência para Idosos (ILPI) [Internet]. Brasília, DF: ANVISA; 2020 [acesso em 01 out. 2020]. Nota Técnica GVIMS/GGTES/ANVISA N 05/2020. Disponível em: https://www20.anvisa.gov. br/segurancadopaciente/index.php/alertas/item/nota- tecnica-n-05-2020-gvims-ggtes-anvisa-orientacoespara-a-prevencao-e-o-controle-de-infeccoes-pelonovo-coronavirus-sars-cov-2-ilpi

34. Brasil. Ministério da Saúde, Secretaria de Atenção Primária à Saúde.. Prevenção e controle de infecções pelo novo Coronavírus (SARS-CoV-2) a serem tratados nas Instituições de Longa Permanência para Idosos (ILPI) [Internet]. 2020 [acesso em 01 out. 2020]. Nota Técnica no 8/2020-COSAPI/CGCIVI/DAPES/SAPS/

MS. Disponível em: http://189.28.128.100/ dab/docs/portaldab/documentos/ notatecnica82020COSAPICGCIVIDAPESSA PSMS02abr2020COVID-19.pdf

35. Brasil. Ministério da Saúde, Secretaria de Atenção Primária à Saúde. Isolamento para Idosos Institucionalizados: orientações de higiene e cuidados [Internet]. 2020 [acesso em 01 out. 2020]. Nota Técnica n 9/2020-COSAPI/CGCIVI/DAPES/ SAPS/MS. Disponível em: http://189.28.128.100/dab/ docs/portaldab/documentos/NT_ANEXO_9_2020_ COSAPI_CGCIVI_DAPES_SAPS_MS.pdf 